\title{
Distribution of the Mandibular Incisor-Mandibular Plane Angle in Nubian Schoolchildren
}

\author{
JAMES E. HARRIS, CHARLES J. KOWALSKI, and SUSAN J. WALKER \\ Department of Orthodontics and Dental Research Institute, University of \\ Michigan, Ann Arbor, Michigan 48104, USA
}

In an earlier paper ( $J$ Dent Res 50:984, 1971) we reported on the distribution of the mandibular incisor-mandibular plane angle by age and sex in a large sample of normal American Caucasian schoolchildren. We found no evidence of sexual dimorphism or of a significant trend with age, and the overall mean value of this measurement was of the order of $97^{\circ}$, ranging from $90^{\circ}$ to approximately $120^{\circ}$. Our find ings differed substantially from those, based on smaller sample sizes, of Downs (Am J Orthodont 34: 812, 1948), Margolis (Am J Orthodont 29: 571, 1943), Noyes, Rushing, and Sims (Angle Orthodont 13: 60, 1943) and Speidel and Stoner (Am J Orthodont 35: 536, 1944) who agreed on a mean value of near $90^{\circ}$ and a range of variation from, roughly, $82^{\circ}$ to $98^{\circ}$; but this difference was attributed primarily to the extent of the discrepancy between the sampled populations, namely, children having normal and excellent occlusions, respectively. However, it also was recognized that any norm or "ideal" value for this measurement might have to be modified by factors other than age, sex, and occlusal pattern, for example, race, the values of other dentofacial measurements, and individual variations within these and other groubings.

We investigated a potential racial difference; we studied the distribution of this measurement in a sample of Nubian schoolchildren, from 6 to 18 years of age, collected as part of our study

This work was supported, in part, by National Institutes of Health Grant No. 03-011-1.

Received for publication July 5, 1974.

Accepted for publication November 4, 1974. of the craniofacial morphology of ancient and modern Nubians (Am J Orthodont 58:578, 1970.

The descriptive statistics for the distribution of the mandibular incisor-mandibular plane angle by age and sex in the Nubian sample are given in the table. The $P$ values for $t$ tests comparing the observed male and female mean values indicate that there is no evidence of sexual dimorphism and no consistent developmental pattern; this suggests that we may pool the sample by sex and age and obtain an overall mean value of the order of $95^{\circ}$ and a range of variation from $82^{\circ}$ to $110^{\circ}$. It is clear that while a considerable overlap of these distributions exists, the distribution for the Nubian schoolchildren lies somewhere in between those found for this measurement by Downs et al $\left(82^{\circ}\right.$ to $\left.120^{\circ}\right)$ and those of our earlier study of American Caucasian schoolchildren $\left(90^{\circ}\right.$ to $\left.120^{\circ}\right)$. Thus, although a comparison with Caucasian standards computed from children having excellent occlusions reveals a higher degree of bimaxillary protrusion among the Nubians, the difference between these groups is less than that exhibited within the Caucasian samples. Thus the observed racial difference is less than that which exists between children of the same race having normal and excellent occlusions. Note, however, that racial differences are a multivariate phenomenon (Kowalski, $A m J$ Phys Anthropol 36: 119, 1972), reflected primarily in differing combinations of dentofacial measurements, which may have to be considered to adequately characterize and distinguish between these groups.

Distribution of the Mandibular Incisor-Mandibular Plane Angle in a Sample of Nubian Schoolchildren

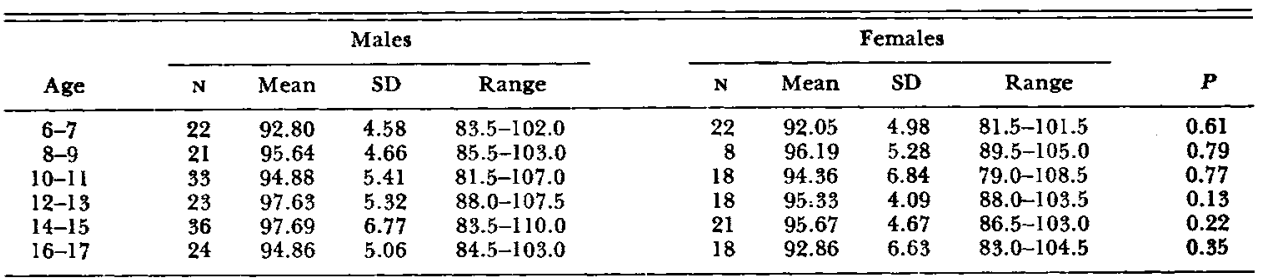

Note: $\mathrm{SD}$, standard deviation. $P, P$ value for $t$ test comparing males and females. 